\title{
Organizational and Individual Safety Factors in Work on Chemical Tanker Vessels
}

\author{
Line Raknes Hjellvik ${ }^{1,2}$, Randi Elisabeth Hope Aga ${ }^{1,3}$ \& Bjørn Sætrevik,1,4
}

\begin{abstract}
Chemical tanker vessels are at risk for large-scale accidents due to the nature of their cargo and operating conditions, challenging environments as well as general maritime hazards. To counteract such hazards, the ship-owning company works on maintaining safety at the organizational level, and the captain instantiates safety regulations on the interpersonal level. The crew members are expected to maintain safety by having accurate situation awareness, and beneficial safety attitudes and behaviour. We pre-registered an analysis to test for associations between safety variables in a survey for chemical tanker vessel crews. A structural equation model revealed that the ship-owning company's safety climate and the captain's leadership style were associated with the vessel's safety climate. Further, the vessel's safety climate was associated with individual safety attitude, situation awareness and adherence to safety management systems. Safety attitude had a central role in the model and was associated with situation awareness, reporting attitude, safe behaviour and adherence to safety management systems. The results imply that it may be beneficial to monitor and improve safety attitudes among crew on chemical tanker vessels and in similar work-environments.
\end{abstract}

\section{Keywords}

Chemical tankers, safety climate, authentic leadership, safety attitudes, situation awareness, structural equation modelling, preregistered study

\section{1: Introduction}

Maritime operations are inherently hazardous (Roberts, 2002). High-risk events such as groundings, collisions, capsizing, crush injuries and falling accidents are some of the dangers associated with maritime vessels (Norwegian Maritime Authority, 2017). Chemical tanker vessels have additional safety challenges as their cargo pose risks of fire, explosion, poisoning and environmental pollution (Celik, 2010). In addition, human error during chemical tanker operations such as tank cleaning, mooring and bulk operations can be fatal. Chemical tanker operations often take place in remote and inhospitable environments such as arctic waters, which may increase both the likelihood and the consequences of accidents. Further, the crew have extensive working hours and long and isolated working shifts with limited contact with family and friends outside of the crew, which may lead to fatigue, boredom and reduced motivation. The crew often have diverse countries of origin, which could cause challenges to safety culture and communication.

Maintaining safety in high-risk organizations requires a thorough understanding of complex associations between psychological factors and safety behaviour. A "systems approach" to risk management (Reason, 1990) involves designing a resilient system that can tolerate and manage errors and operational dangers. In addition to structural and technical safety barriers, both organizational and individual factors such as leadership, situation awareness and safety attitudes may lead the crew to become sufficiently knowledgeable and vigilant about risks in their workenvironment, and to behave in compliance with formal and informal safety expectations. We therefore expect that the safety climate on the company and on the vessel level, the captain's authentic leadership style, the crew member's situation awareness and safety attitudes may function as safety barriers on chemical tanker vessels.

\section{1: Psychological safety factors}

1.1.1: Safety climate.The safety climate reflects and expresses the core values of the organizational culture (Mearns, Flin, Gordon, \& Fleming, 1998; Guldenmund, 2000). Safety climate is an expression of the current level of the more general safety culture (Flin, Mearns, Connor, \& Bryden, 2000), and occurs in the social and psychological dynamics of the employee's assessment of safety. These perceptions of safety-related elements constitute a frame of reference for expected behaviour within the organization. Thus, safety climate can be defined as shared perceptions related to safety in the workplace (Zohar, 1980). Safety climate is considered to be a critical factor for safety in high reliable organizations, and it has been associated with several individual and organizational factors, such as health problems, job satisfaction, intentions to leave the job, leadership, risk perception (Nielsen, Eid, Hystad, Sætrevik, \& Saus, 2013), safety behaviour (Lu \& Tsai, 2010), safety participation and compliance (Clarke, 2006). Multiple levels of safety climate within the same organization may lead to discrepancy between employee's perception of safety climate in the organization and in different subunits, e.g. that the organization's focus on safety may differ from the subunit's focus on safety (Zohar \& Luria, 2005). For vessels that are part of a larger organization, we may differentiate between the safety climate for the vessel, created by actions and attitudes of the vessel's officers, and the safety climate for the shipowning company, created by company-level regulations and safety campaigns. In the current study, safety climate perceptions are related to competing requirements and

\footnotetext{
${ }^{1}$ Operational Psychology Research Group, Faculty of Psychology,

University of Bergen, Christies gate 12, NO-5015 Bergen.

${ }^{2}$ Email: line.hjellvik@uib.no, corresponding author

${ }^{3}$ Email: randielisabethaga@gmail.com

${ }^{4}$ Email: bjorn.satrevik@uib.no
} 
consistency and non-conformity between guidelines, procedures and decisions (Zohar, 2010) in the company and on the vessels. An organization or vessel with a healthy safety climate is expected to have a positive influence on individual safety attitudes in that these perceptions of expected behaviour is transferred to individual beliefs. Similarly, vessels that prioritize safety are expected to have employees who use the safety management system as intended and take responsibility for maintaining an accurate situation awareness of safety aspects in their work.

1.1.2: Authentic leadership.The extent to which a leader emphasizes safety may affect employee's safety behaviour, and leaders that are committed to building good relationships with employees could have severe impact on safety culture issues. Authentic leadership (AL) is a leader-follower relationship that can lead to positive organizational outcomes through the leader's behaviour acting as a role model for followers (Gardner, Avolio, Luthans, May, \& Walumbwa, 2005). Walumbwa and colleagues (2008) suggested that the construct of AL includes four core components: 1) Balanced processing of information, 2) Relational transparency, 3) an Integrated moral perspective and 4) Self-awareness. Leaders who listen to others, are honest, have high moral standards that are not easily changed by external factors as well as sufficient self-insight may contribute to a safer work environment. Sætrevik and Hystad (2017) found associations between AL and situation awareness, as well as between AL and admitting to committing unsafe actions. Further, a positive association has been observed between AL and employees' perception of the safety climate on board cargo vessels, and among workers on offshore oil and gas installations (Borgersen, Hystad, Larsson, \& Eid, 2014). Leaders who exhibit an AL style may contribute to several benefits such as job satisfaction (Černe, Dimovski, Marič, Penger, \& Škerlavaj, 2014). It is reasonable to assume that the captain's leadership may contribute to a sound workenvironment and a beneficial safety climate on the vessels. An authentic captain may support and motivate the crew's safety behaviour, and the captain's behaviour may serve as a model for the crew's own behaviour. The captain's leadership may thus facilitate individual situation awareness, safety attitudes and behaviour.

1.1.3: Situation awareness. Whether a crew member behaves safely in a situation relies on their cognitive assessment of their current environment and associated risks. Situation awareness (SA) represents an individual's understanding of the environment and is a result of perceiving and integrating relevant information which facilitates prediction of future events. Endsley (1995) argued that an operator needs to have accurate SA to perform tasks in an effective and safe manner. $\mathrm{SA}$ is seen as an antecedent in the decision-making process, and an accurate SA will facilitate appropriate responses and decisions which prevent negative outcomes. There are several individual and situational factors that may facilitate or hinder the development of SA. Workplace design, workload, collaboration, stress, competence, experience, confidence and attention are some of the factors that could impact how precise an operator is able to perceive, comprehend and anticipate dynamic elements in the environment. A work environment that is organized and designed in accordance with human abilities and cognition may facilitate SA. The relationship between subjective experience and objective reality, and the three-part conceptualization of SA have been questioned and discussed by Dekker, Hummerdal \& Smith (2010). However, such a tripartite division has proven to be relevant in an applied setting (Sneddon, Mearns, \& Flin, 2006). Both objective and subjective methods have been used to measure the construct (e.g. Salmon, Stanton, Walker, \& Green, 2006; Salmon, Stanton, Walker, Jenkins, Ladva, Rafferty, \& Young, 2009). Edgar and colleagues (2018) made a similar distinction between "actual SA" and "perceived SA", in which the former is the individual's assessment compared to an objective truth, and the latter represents how an individual perceives his or her own SA. Sætrevik and Hystad (2017) used a context general inventory to measure respondents' subjective assessment of their own SA across work tasks onboard offshore supply vessels. The results showed that higher levels of reported SA were associated with lower levels of reported unsafe actions and subjective risk assessment. Those of the crew who experience that they are able to monitor safety cues, understand the safety aspects of the systems they operate in, and predict which safety scenarios that may develop over time, may also be able to perform their work safely.

1.1.4: The role of attitudes in safety.Safety climate, leadership style and SA have been considered to be vital for determining a crew member's safe conduct (Dahl, Fenstad, \& Kongsvik, 2014; Sætrevik \& Hystad, 2017). In addition, a crew member's attitudes about safety may modulate their safe behaviour. Attitudes are typically considered as being comprised of affective evaluations and cognitions about an object (Crano \& Prislin, 2006). Attitudes to a specific behaviour correlates with the performance of that behaviour (Ajzen \& Fishbein, 1973), which suggests that the safety attitudes of the crew on chemical tanker vessels should predict their safety behaviour. Employee safety attitudes are formed by both individual and situational factors and will be influenced by the extent to which the employee perceives the company to prioritise safety (Mearns \& Flin, 1995). Further, the safety attitude may impact beliefs and knowledge about factors that influence risk and safety in the workplace, motivation to adhere to safety regulations, and perseverance in following procedures.

Routines for employees to report unwanted incidents or conditions are typically an extensive part of safety management systems in settings such as chemical tanker vessels. However, there is some uncertainty about how such routines and instructions to report are understood by the crew, how they are used (Lappalainen, Vepsäläinen, Salmi, \& Tapaninen, 2011; Storgård, Erdogan, Lappalainen, \& Tapaninen, 2012) and their relation to actual incidents. Crew members do not typically have clear incentives for reporting, there are opportunity costs to reporting, and they may fear that the reporting has negative consequences. Therefore, the attitudes that the crew members have to reporting systems may be important for whether the reports can be trusted as reliable indicators of the safety level. To our knowledge, there are no existing measures for reporting attitudes in the maritime industry. A measure of attitudes to reporting is currently under development across three surveys (see preregistration Sætrevik \& Hjellvik, 2017).

1.1.5: Safety outcomes. It is challenging to find reliable objective measures for workplace safety, as accidents 
(particularly high consequence accidents) are infrequent and observations of "near misses" may be unreliable. Direct measures of safety compliance are resource intensive and difficult to scale in applied settings. As a proxy for the objective safety level, one may ask operators to report their degree of compliance to safety regulations and to which extent they avoid committing unsafe actions. This would be actions such as letting their choices favour safety rather than efficiency, warning colleagues about possible dangers or avoid parallel work operations that could lead to dangerous situations. Several studies have revealed an association between unsafe behaviour and accidents (Hobbs \& Williamson, 2002; Hofmann \& Stetzer, 1996; Tomás, Cheyne, \& Oliver, 2011).

In addition to safety behaviour, the self-reported use of the safety management system could be an indicator for safety. Safety management systems are various procedures and practises designed to guide safe behaviour so that unwanted incidents and accidents are avoided. Examples of compliance to safety management systems is the extent to which various safety procedures for work on board are followed, how safety responsibilities are administered and managed, to which extent rest hour regulations are adhered to, and whether time is set aside to perform administrative safety work.

The reliability of self-report depends on the operators' ability and motivation to report accurately. While social desirability and self-serving bias may have some influence on the reporting of issues where there is a clearly preferred attitude, most employers would also expect operators to admit variability in how they relate to complex safety issues in their day-to-day work.

\section{2: Current study}

1.2.1: Research needs. As shown in the review above, vessel and company safety climate, AL, SA, safety and reporting attitudes are individual and organizational factors that have been suggested as predictors of maritime safety. However, there is a need to describe the relationship between such factors and to investigate to which extent they are relevant for safety on chemical tanker vessels.

The current study was conducted as an unpaid research collaboration with an industry partner to investigate the safety culture among crew on chemical tanker vessels in Northern Europe. The aim was to identify variables that are relevant for safety on chemical tanker vessels, the relationships between them, and their relative contributions. The study also takes some steps in developing tools for company-wide measurement of safety and reporting attitudes, and safety compliance.

1.2.2: Hypotheses.Based on the discussion above, we expect that the company and the captain's efforts to emphasize and support safety will be reflected in the crew members' evaluation of the safety climate in the organization, and to be one of the factors that contribute to the safety climate on the vessels. We thus expect "Company SC" and "Vessel SC" to be positively associated (H1). An AL style among the vessel captains may involve beneficial interactions that facilitates a work-environment that emphasizes safety and inspires and validates the crew members' perceptions, thoughts, beliefs, feelings and commitment to safety. Hence, we expect "AL" to be positively associated with "Vessel SC" (H2a), with "Safety attitude" (H2b), with "SA" (H2c), and with "Reporting attitude" (H2d). Further, the safety values and attitudes expressed by the captain and others on the vessel may impact the crew's safety attitudes, behaviour and compliance to regulations. Hence, we expect the "Vessel SC" to be positively associated with "Safety attitude" (H3a), with "SA" (H3b), with "Safety management system" (H3c), and with "Reporting attitude" (H3d). Further, we expect that crewmembers who exhibit positive attitudes to safety also will be more careful and observant about safety in their work, be motivated to work in accordance with the safety management system and notice and be motivated to report deviations from safety regulations. Hence, we expect "Safety attitude" to be positively associated with "SA" (H4a), with "Safe behaviour" (H4b), with "Safety management system" (H4c), and with "Reporting attitude" (H4d). Finally, we expect that crew who report that they notice safety cues, understand the overall situation, and are able to predict the development of a safety situation, will also report more compliance to safety regulations. Hence, we expect SA to be positively associated with "Safe behaviour" (H5). All hypotheses are shown visually in Figure 1

1.2.3: Preregistration. The study was pre-registered on the Open Science Framework before data collection. The registration describes the hypotheses, data collection, and analysis approach (https://osf.io/cdh5p). Pre-registration of studies is meant to ensure transparency, limit questionable research practices and contribute to a more rigorous scientific process in psychology. Such research designs are intended to counteract some of the factors that have contributed to the current replication crisis in psychology (see e.g. Wagenmakers, Wetzels, Borsboom, van der Maas, \& Kievit, 2012).

\section{2: Methods}

\section{1: Measurement}

2.1.1: Data collection. A survey of various safety variables was sent out to all crew working in a Norwegian ship-owning company. Responses were collected from December 2017 until April 2018. Eighteen chemical tanker vessels participated, and as vessel manning varies between 30-35 employees, the population was approximately 450 respondents. Participation was not compensated but was encouraged by both company and vessel management.

The first page of the survey provided informed consent and conditions for participating. After seven items measuring demographic and employment information, there were 115 statements about SA, safety climate, AL and safety leadership, safe behaviour, safety management system and safety attitudes. The respondents indicated the extent to which they agreed to the statements on a 5-point Likert scale, where the extremes were labelled as "completely disagree" and "completely agree". To control for response bias there were both positively and negatively phrased items. On the suggestion of our collaborators in ship-owning company, we rephrased some of the items to make them more accessible for crew members with English as a second language. A coding system between the researchers and the ship-owning company ensured that researchers did not know which vessel 
the returned surveys came from. The study was approved by the Norwegian Centre for Research Data (project 56912).

The crews returned 328 surveys, yielding a response rate of about $73 \%$. Three of the surveys were blank, four surveys had used the same response options for all items, and seven surveys indicated non-compliance or lack of language understanding, such as disregarding negative phrasing of statements. These fourteen surveys were removed from the sample. In addition, one respondent had declined to fill out the pages for safety climate and AL and was not included in those calculations and analyses. Finally, 21 surveys from the captains were excluded, in accordance with the pre-registered analysis plan. The resulting sample for analysis was 292 responses.

2.1.2: Variables. "Safety climate" was measured with a scale developed by Zohar and Luria (2005), where crew members' perceptions of considerations for safety are measured both on the level of their immediate work-place provided by their supervisor, and by their more general workconditions provided by the company management. 16 items (37-52) were used to measure the safety climate in the shipowning company (Company SC), and a sample item is: "The ship-owning company reacts quickly to solve the problem when told about safety hazards". 16 items (21-36) were used to measure the safety climate aboard the vessels (Vessel SC), and a sample item is: "The captain makes sure we receive all the equipment needed to do the job safely". Cronbach's alpha was .95 for Company SC and .936 for Vessel SC. For both levels of safety climate, an exploratory factor analysis was performed, fixed to extract three factors with a maximumlikelihood estimation. Based on this, three item parcels were made based on item factor loading to be used as indicators for the latent variables in the SEM analysis.

AL was measured with a 16-item scale (53-68) developed by Walumbwa and colleagues (2008). The scale comprises different statements about leadership, and these statements applies to the captain in the current setting, such as: "The captain seeks feedback to improve interaction with others", "The captain says exactly what he means, "The captain ask others to present views that challenges his views" and "The captain demonstrate beliefs that are consistent with actions ("walk the talk."). Cronbach's alpha was .935. The scale can be decomposed into four core components: self-awareness (65-68), relational transparency (53-57), balanced processing (62-64) and internalized moral perspective (58-61). This division into four components will be used as indicators for the latent variable in the SEM analysis.

"Safety attitude" was measured with 13 items (numbered 100-101,112-122). Some of the items were taken from previous measures (e.g. Rundmo, 1994), and from discussions between researchers and industry partners. Some of the items were inspired by the ship-owning company's current HSE campaign. From a theoretical analysis of item content, nine of the items were classified as relating to "safety motivation", with item text such as "It is acceptable for me to take chances if I am the only person at risk", while the remaining four items were related to "safety prioritization", with item text such as "Safety procedures often stand in the way of getting the job done efficiently." These two sub-categorizations will be used as indicators for the latent variable in the SEM analysis. Cronbach's alpha for the safety attitude scale was .7.
"Reporting attitude" was measured with 12 items (numbered 84, 93, 102 - 111). Eight of the items (inspired by Probst \& Graso, 2013, such as "Since it's impossible to prevent all accidents/near-misses, we shouldn't spend too much resources on reporting") were related to the assumed consequences of reporting, while the remaining three items were related to the motivation and use of reporting system (with items such as "I'm not motivated to report accidents/near-misses"). The division was used to make two indicators for the latent variable in the SEM analysis. The items have been developed across three previous survey data collections (see pre-registration Sætrevik \& Hjellvik, 2017), where item text was suggested and modified based on input and feedback from subject matter experts and industry collaborators, and response distributions from offshore maritime employees. Cronbach's alpha was .878 .

SA was measured with a context general 13-item scale developed by Sætrevik (2013). The items were based on Endsley's (1995) theoretical conceptualization of SA which has three levels of cognitive information processing. Perception (level 1) was measured with items 9, 11, 17 and 19, such as "I sometimes lose track of information relevant for maintaining safety in my work". Comprehension (level 2) was measured with items 10,13,15, 16 and 20, such as "It can be difficult to know which consequences my actions will have for safety". Projection of future events (level 3) was measured with items 8, 12,14 and 18, such as "I notice when an unsafe situation is about to arise at my workplace". Cronbach's alpha for the reporting attitude scale was .811. The division of items into three levels will be used as indicators for the latent variable in the SEM analysis.

"Safe behaviour" was measured with seven items (numbered 74-80) taken from the safety literature (Nielsen, Hystad, Sætrevik, \& Saus, 2013), and items developed by the researchers in collaboration with our industry partners. The items measured to what extent the participants felt that they were working safely. A sample item is: "I stop work if I think it can be dangerous for me or others to continue". Cronbach's alpha for the "safe behaviour" items was .7. The items will be combined to an average score to be used in the SEM analysis.

"Safety management system" was measured with 17 items (numbered 81-83, 85-92, 94-99). Some of the items (88$92,95-97)$ were related to the respondent's self-reported compliance to the safety management system, while items 81 $83,85-87,98$ were related to the respondent's impression of the extent to which the vessel's crew complied to the safety management system. A sample item is: "Insufficient maintenance has led to poorer safety". Cronbach's alpha of the items was 7. The items will be combined to an average score to be used in the SEM analysis.

\section{2: Analyses}

In accordance with the pre-registration and as described in section 1.2.2, we presented five main hypotheses, of which three had four sub-divisions. The hypotheses described relationships between the variables AL, Safety climate (company and vessel level), SA, Safety and Reporting attitudes, and indexes for Safe behaviour and Safety management system. A SEM model was constructed to reflect the hypotheses H1, H2a-d, H3a-d, H4a-d and H5, shown in Figure 1. The model was tested in STATA using the 
gsem package. The analysis followed the pre-registered analysis plan, but an additional revised SEM model was performed based on the results from the pre-registered model.

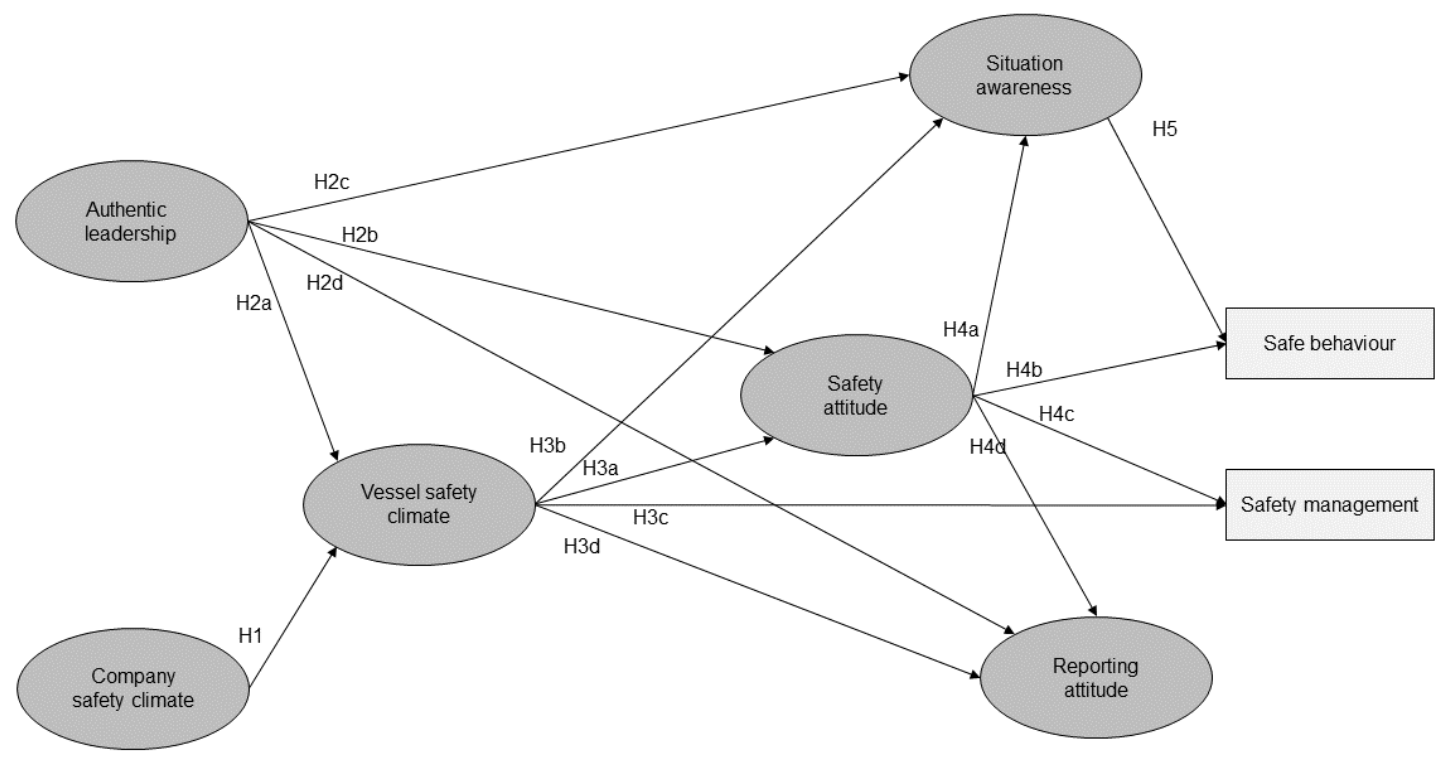

Figure 1: The pre-registered hypothesis model depicting the expected positive associations (H1 - H5) between the predictors and safety outcomes.

\section{3: Results}

\section{1: Description of Sample and Measured Variables}

The analysis sample consisted of 328 respondents (see section 2.1.2), from 18 different vessels, with 11-28 responders from each vessel (median $=14)$. Of those that had stated their nationality $75 \%$ were Filipino and 17\% were Latvian, while the remaining were Russian, Norwegian, Lithuanian and "other" (76 missing responses). Most (59\%) worked on deck, $31 \%$ in the engine room, and $10 \%$ in the galley. Although $60 \%$ had been working for the ship-owning company for five years or more, half $(49 \%)$ had been working on the current vessel for less than 1 year, and $77 \%$ for less than three years, which indicates that crew members change between working on different vessels. Average scores were lower for negatively phrased items, which may indicate that some respondents were confused by the reversed phrasing. However, to be able to compare with other studies using the same measures, we chose to retain all items in our variables. Challenges to validity due to language issues are further discussed below.

We will describe average responses and distributions for the variables in the survey below. In some cases, the current average values may be compared to a Norwegian maritime sample of 1,828 crew members working on offshore hydrocarbon production support vessels (Sætrevik, Ørbeck, Helland \& Eid, 2019).

The average value of "Company SC" in our sample was $4.34(\mathrm{SD}=0.6)$, and "Vessel SC" had a similar average $(\mathrm{M}=$ $4.37, \mathrm{SD}=0.57)$. This indicates that the crew members responded that they more than agreed that the ship-owning company and the vessel management provided a safe workenvironment. The current values are higher than what was reported in the offshore sample (company SC M $=4.07, \mathrm{SD}$ $=0.71$, vessel $\mathrm{SC} \mathrm{M}=4.28, \mathrm{SD}=0.67$ ).

The mean value of "AL" was $4.08(\mathrm{SD}=0.6)$, indicating that the crew on average reported that they saw their captain as authentic. In the offshore sample, average AL was slightly lower, at $3.92(\mathrm{SD}=0.67)$.

Average "Safety attitude" was $4(\mathrm{SD}=0.48)$, and average "Reporting attitude" was $3.94(\mathrm{SD}=0.76)$ indicating that the crew agreed to positive statements that they give priority to safety and see the value of reporting. These values are somewhat higher than in the offshore sample ("Safety attitude" $\mathrm{M}=3.92, \mathrm{SD}=.73$, "Reporting attitude" $\mathrm{M}=3.78$, $\mathrm{SD}=.83$ ), although a direct comparison is difficult, as the item count and content are different for these variables.

Average score on "SA" was $3.95(\mathrm{SD}=0.56)$, indicating that the crew on average agreed that they are aware of safety critical cues in their environment. These values are somewhat higher than the values in the offshore sample $(\mathrm{M}=3.79$, SD $=0.58)$.

Average response on the "Safe behaviour" items was $3.95(\mathrm{SD}=0.73)$ and "Safety management system" was 3.87 $(\mathrm{SD}=0.48)$. This indicates that the crew agreed that they perform their work safely and that safety tools are used in the way they are meant to be used. Comparing these to similar measures with some of the same items in the offshore sample showed similar value for "Safe behaviour" $(\mathrm{M}=3.91, \mathrm{SD}=$ $0.86)$, but somewhat lower values for "Safety management system" $(\mathrm{M}=4.04, \mathrm{SD}=0.53)$.

\section{2: Structural Equation Modelling}

3.2.1: Pre-registered model. A confirmatory factor analysis (CFA) of the pre-registered model (covariates between latent variables and paths to the observed variables) showed moderate-to-good model fit $\left(\mathrm{x}^{2}(135)=341\left(\mathrm{x}^{2} / \mathrm{df}=2.53\right)\right.$, RMSEA $=0.073$ (with $90 \% \mathrm{CI}=.064-.083$ ), SRMR $=.051$, TLI $=0.936)$. Compared to fit index guidelines ( $\mathrm{Hu} \&$ Bentler, 1999), the pre-registered model may be interpreted as having moderate-to-good fit (all indices were within acceptable ranges, except for RMSEA that was slightly above the suggested cut-off value of .06). 
The SEM of the pre-registered model showed moderateto-good model fit. Nine of the fourteen associations between latent variables in the model were significant, with beta coefficients ranging from .13 to .91 . The pre-registered model explained $\mathrm{r}^{2}=.74$ of the "Vessel SC" variance, .68 for "Situation awareness", .25 for "Safety attitude", and .85 for "Reporting attitude". All results, fit indices and a figure for the pre-registered SEM model are available as additional online materials in the OSF folder online https://osf.io/rg598/ and are discussed below.

3.2.2: Revised model. Based on the results of the preregistered SEM, we prepared a revised model shown in Figure 2 below where we removed non-significant associations $\mathrm{H} 2 \mathrm{~b}$, $\mathrm{H} 2 \mathrm{c}, \mathrm{H} 2 \mathrm{~d}, \mathrm{H} 3 \mathrm{c}, \mathrm{H} 3 \mathrm{~d}$ and $\mathrm{H} 5$. As both $\mathrm{H} 2 \mathrm{c}$ and $\mathrm{H} 3 \mathrm{~b}$ relate to how the captain's leadership influences crew member SA, they are likely to account for overlapping portions of the variance. $\mathrm{H} 3 \mathrm{~b}$ was significant with one-tailed testing (in accordance with the pre-registration). We thus retain $\mathrm{H} 3 \mathrm{~b}$ in the revised model, to test for the captain's influence on the crew's SA. A CFA of this revised model showed moderateto-good model fit $\left(\mathrm{x}^{2}(136)=342\left(\mathrm{x}^{2} / \mathrm{df}=2.51\right), \mathrm{RMSEA}=\right.$ 0.073 (with $90 \% \mathrm{CI}=.063-.0 .83$ ), SRMR $=.052$, TLI $=$ 0.936).

The revised structural model (see Figure 2) explained $\mathrm{r}^{2}$ $=.72$ of the "Vessel SC" variance, .67 for "SA", .24 for "Safety attitude", and .86 for "Reporting attitude". The results of the pre-registered and the revised model are discussed in relation to all the pre-registered hypotheses below.

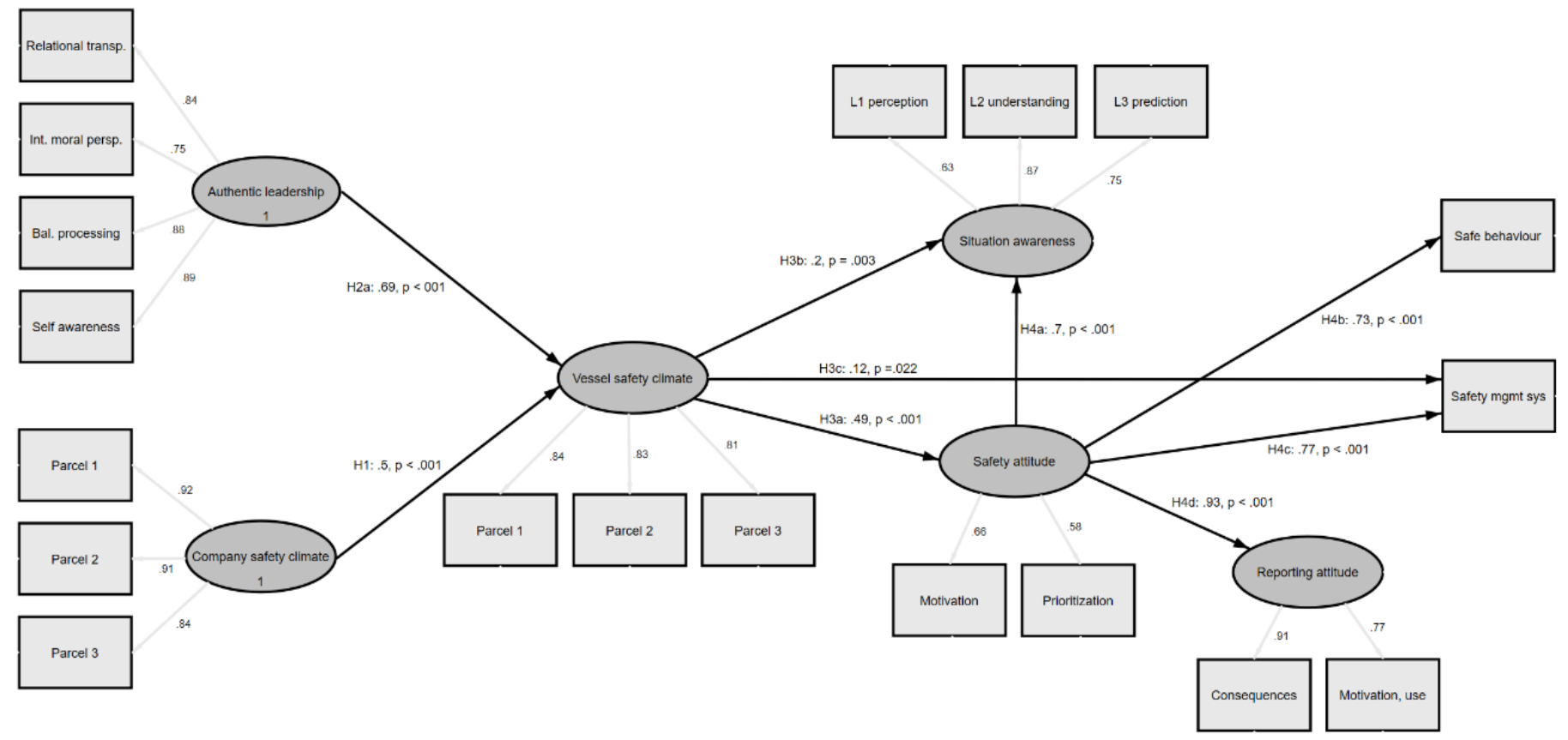

Figure 2: Results from the revised structural model showing standardized estimates for all five bypotheses. Coefficients are marked with bypothesis number and two-tailed p-values. Error terms and observed indicators for the latent variables are left out of the figure for ease of reading. Fit indices indicate moderate-to-good fit for most indices $(x 2(145)=498(x 2 / d f=3.44), R M S E A=0.093$ (with 90\% CI = .084-.102), TLI =0.897, but note SRMR $=.186$

3.2.3: Support for H1. "Company SC" was positively associated with "Vessel SC" (H1). This means that crew members who perceived the ship-owning company to make provision to maintain safety also perceived the captain to maintain safety on the vessel.

3.2.4: Support for H2. The captain's "AL" was positively associated with "Vessel SC" (H2a), showing that crew members who perceived their captains as authentic leaders also had positive impressions of the safety climate that the captain creates on the vessel. "Company SC" and "AL" accounted for $22 \%$ of the variation in "Vessel SC". Three associations in the pre-registered model were not supported, in that "AL" was not associated with "Safety attitude" (H2b, $\mathrm{p}=.18), \mathrm{SA}(\mathrm{H} 2 \mathrm{c}, \mathrm{p}=.92)$ or "Reporting attitude" (H2d, p $=.13)$.

3.2.5: Support for H3 The "Vessel SC" had a positive association with "Safety attitude" (H3a), with "SA" (H3b) and with "Safety management system" (H3c) in the revised model. This implies that crew who perceived the safety climate aboard the vessels to be positive also had positive attitudes to safety, an accurate SA and proper safety management. One of the associations in the pre-registered model was not supported, in that "Vessel SC" was not associated with "Reporting attitude" (H3d, p = .5).

3.2.6: Support for H4. The crew member's "Safety attitude" was positively associated with "SA" (H4a), with "Safe behaviour" (H4b), with "Safety management system" (H4c), and with "Reporting attitude" (H4d). All preregistered $\mathrm{H} 4$ hypotheses were thus supported. This indicates that crew who reported a positive attitude to safety also reported a positive attitude to reporting practises. Crew who reported a positive attitude toward safety also reported that they perform their work safely, and that they use the safety tools in accordance with the safety management system.

3.2.7: Support for $H 5$. The pre-registered association between "SA" and "Safe behaviour" was not supported (H5, $\mathrm{p}=.33$ ), and was not included in the revised model. This indicates that crew who reported that they have a good SA 
did not systematically report that they perform their work in a safe manner

\section{4: Discussion}

\section{1: Summary of results}

The aim of this study was to test if various organizational and individual factors impact the safety behaviour of crew on chemical tanker vessels. The hypotheses described positive associations between "Company SC" and "Vessel SC" (H1), between "AL" and "Vessel SC" (H2a), "Safety attitude" (H2b), "SA" (H2c) and "Reporting attitude" (H2d), between "Vessel SC" and "Safety attitude" (H3a), "SA" (H3b), "Safety management system" (H3c) and "Reporting attitude" (H3d), between "Safety attitude" and "SA" (H4a), "Safe behaviour" (H4b), "Safety management system" (H4c) and "Reporting attitude" (H4d) and between "SA" and "Safe behaviour" (H5).

Nine of the fourteen associations were significant in the pre-registered model. Based on these results and a theoretical review, we tested a revised, more parsimonious model where we removed the non-significant associations: H2b-c and H3d. The revised model had overall moderate to good fit. The test of the pre-registered hypotheses suggests that "Company SC" and AL both have a direct impact on "Vessel SC", which in turn has a direct impact on "Situation awareness", "Safety attitude" and "Safety management system". "Safety attitude" has a direct impact on SA, "Reporting attitude" and both outcome measures. This indicates that "Company SC" and AL have an indirect impact on safety, first through "Vessel SC", and then through "Safety attitude". This supports the idea that organizational factors impact individual factors which in turn impact safety behaviour. All pre-registered associations are further discussed below.

"Company SC" and AL were associated with "Vessel SC" (H1 and H2a) which indicates that crew who think that the ship-owning company supports safety initiatives, and see their captains as authentic, also think that there is a safety conscious work-environment on the vessel. This result is in line with work by Zohar and Luria (2005) that showed a correlation between organization climate and group climate, and work by Borgersen and colleagues (2014) and Hystad, Bartone, and Eid (2014) that showed AL to be associated with safety climate. "Company SC" and "Vessel SC" measure the same theoretical construct on two organizational levels and are designed to have similarly phrased items in order to enable comparison between the different levels. This may account for some of the association between the variables. "Vessel SC" was associated with "Safety attitude" (H3a), indicating that crew members who think that the captains give priority to safety in their provisions for the vessel's workenvironment also report that they have attitudes beneficial for safety. "Vessel SC" was further associated with "SA" (H3b) which supports the idea that crew members who see safety as being a priority on the vessel also state that they are aware of safety cues, can build an understanding of the safety situation and anticipate future safety outcomes of their actions. "Vessel SC" was also associated with "Safety management system" $(\mathrm{H} 3 \mathrm{c})$ indicating that crew who experience a positive safety climate on the vessels also state that they use the safety tools in line with the safety management system. The preregistered model assumed that both "AL" and "Vessel SC" would be associated with "SA" (H2c and H3b, respectively), but found neither to be significant. However, as both these predictors concern how the captain is seen as a safety leader, one may expect the explained variance of the two factors to overlap. As the revised model removes one of the associations ( $\mathrm{H} 2 \mathrm{c})$, the remaining association $(\mathrm{H} 3 \mathrm{~b})$ accounts for more of the variation in situation awareness. However, one should note that the coefficient between "Vessel SC" and "SA" is low in both models.

"Vessel SC" was not associated with "Reporting attitude" (H3d). This means that whether the crew sees safety as being a priority in the work-environment on board was not associated with attitudes to reporting. Further, "AL" was not associated with "Safety attitude" (H2b), "SA" (H2c) or "Reporting attitude" (H2d). This indicates that a more authentic captain was not associated with the crew member's self-reported cognitive states or attitude measures. The lack of support of $\mathrm{H} 2 \mathrm{c}$ is particularly striking, as two previous survey studies using similar measures (Sætrevik \& Hystad, 2017; Sandhåland, Oltedal, Hystad \& Eid, 2017) have shown AL to be associated with SA in maritime samples. A social desirability bias (Edwards, 1953) may have obscured the tests of $\mathrm{H} 3 \mathrm{~d}$ and $\mathrm{H} 2 \mathrm{~b}-\mathrm{d}$. If the crew were concerned with expressing positive safety values when answering the survey, this may have reduced the variation and systematic relationships, and may have made associations more difficult to detect. While social desirability may have influenced several of the measurements, the items related to "AL", and "Vessel SC" might be especially prone to this bias, as these items comprise statements about the crew's superiors.

"Safety attitude" was associated with "SA" (H4a), "Safe behaviour" (H4b), "Safety management system" (H4c) and "Reporting attitude" (H4d) indicating that attitudes promoting safety are related to an increase in safety in cognition and compliance. Thus "Safety attitude" appears to have a central role in the theoretical model, and this variable seems to be a mediating factor between organizational factors and safety outcomes. Finally, there was no association between "SA" and "Safe behaviour" (H5) which indicates that being aware of safety cues and understanding the risk situation did not predict behaviour. This null finding is further discussed below (section 4.2.3.).

\section{2: Mechanisms for Effects}

4.2.1: Organizational influences. In their efforts to comply with regional and international safety standards, to avoid accidents and provide a safe work-environment for their employees, the ship-owning company sets the procedures and safety regulations for the organization and works actively to communicate their expectations to the crew in order for them prioritize safety in their individual work. Zohar (2000) argued that the company's safety prescription must be followed by instrumental rewards for adequate behaviour. In a shipowning company, such rewards could be interpersonal actions such as "saying a good word" to employees who pay attention to safety, or organizational incentives such as investing time and money in safety measures. The perception 
of safety climate is both an individual and a concerted assessment, and these abstract phenomena activates emotional and cognitive mechanisms, which in turn make individuals committed to action (Torner, 2011; Zohar \& Luria, 2005). The crew's perception of the safety climate provides them with informational value about how their superiors expect them to relate to safety. The current results indicate that the quality of the safety climate in the organization has an impact on the safety climate on the vessels. A variety of organizational conditions may contribute in shaping the atmosphere onboard the vessels. The organizational culture, the crew's competence and experience, training and guidance, values, norms and efficiency requirements can be expected to impact the perception of safety climate. The closed milieu aboard the vessels and the hierarchical organization contribute to give the captains an essential role that influence the crew's behaviour. The current results showed that crew members that perceived the ship-owning company to be committed to safety also claimed to behave in a way that supported the vessel's safety. When the crew perceives the ship-owning company's safety philosophy to also be reflected in the extent to which they reward and make provisions for safety in their day-to-day work, a safety conscious climate develops and makes the crew motivated to work safely. Zohar and Luria (2005) suggested that safety climate perceptions are related to commitment, and that the relationship between supervisor and subordinate are connected to leadership-perceptions.

4.2.2: Leadership-follower influences. The captain is seen as the superior authority on maritime vessels, and the crew see the captain as responsible for all decisions on board. On chemical tanker vessels, the captain has daily interactions with the crew and is responsible for communicating and enforcing safety regulations, procedures and priorities. The quality of this interaction shapes the safety climate on the vessels which further impacts individual attitudes and safety behaviour. Walumbwa and colleagues (2008) emphasized that leaders should be aware of how they appear and impact others, consider situations from various perspectives and act in accordance with internal moral standards that displays one's true self (AL). Captains that understand the contextual and individual challenges that the crew face in balancing safety against demands for efficiency may be able to guide them in this, and thus serve as a role model, in addition to having a positive impact on the crew's safety management. Authentic leadership creates a democratic environment where ideas and feedback are appreciated. In the current setting, this would imply that the captain tries to compensate for the hierarchical power distance (Hofstede, 2011) between captain and crew, to allow the crew to make independent safety decisions. The qualities inherent in AL may reduce power distance and improve social interactions. Social interactions of high quality may further promote mutual trust (Torner, 2011), and this reciprocal relationship creates a healthy work-environment which further fosters a positive safety climate.

The chemical tanker vessels in the current study have multicultural crews. As the expectations for leader-follower interactions vary between cultures, it could be that the AL style does not show the same advantages for crews of the current composition. For example, in a culture where display of emotions across power structures is unusual, respondents may interpret authentic qualities such as "The captain shows how he feels" differently from where such variables are typically measured. Such mechanisms may cause the study to find false associations between variables. However, our study indicated that authentic leaders are associated with positive vessel safety climate despite the culturally diverse composition of the crew.

4.2.3: Cognitive factors. The naturalistic decision-making perspective assumes that decision makers use their experience and SA to choose a strategy for further action, rather than comparing different options (Zsambok, 2014). An operator will be able to work safer with a recognition of safety cues, an understanding of how the cues build a coherent situation and an anticipation of how the situation in the work environment will develop over time (SA). Perceiving the current status and an overall understanding of the environment creates a mental model which is used to make appropriate decisions (Endsley, 1995; Endsley, 2000). The crew on chemical tanker vessels face challenging situations such as handling dangerous cargo. This requires an accurate perception of critical cues, as well as a holistic comprehension of what these cues indicate and what further scenarios might occur. Our hypotheses assumed that operators who are aware of critical cues in their current environment would also work in accordance with safety regulations, and to a lesser extent expose themselves or others to unsafe situations. However, in contrast to previous studies using similar measures (Sætrevik \& Hystad, 2017; Sætrevik \& Hystad, 2019) there was no association between SA and safe behaviour in the current dataset. One possible explanation for this inconsistency may be that the current sample answered the survey in their second language. The SA statements may have been particularly complex to understand, as they consisted of both positive and negative semantic content, which may have obfuscated a systematic relationship between $\mathrm{SA}$ and other variables.

4.2.4: Effects of attitudes on safety.Past work in social psychology has established causal relationships between attitudes and behaviour. Ajzen's theory of planned behaviour (1991) states that specific attitudes, subjective norms and perceived control determine an individual's intentions and predict their behaviour. Thus, in an applied and complex setting such as the high-risk work environment of chemical tanker vessels, the relationship should be tested between specific attitudes and specific behaviour. In the current study, the crew reported how they feel and what they think about specific safety issues such as prioritizing safety over efficiency, the culture for blame and the compliance to safety management system, procedures, regulations and reporting. The current results show that safety climate and AL were antecedents for safety attitudes, which further predicted safety behaviour. Supportive, authentic and safety-oriented leaders exert a positive social pressure on the crew's perception of proper safety behaviour. Crew who experience the benefits of the safety culture in the organization, also adopt positive beliefs and thoughts about safety. These aspects may impact performance intentions and influence the crew's commitment and motivation to act safely and conform 
to the safety management system. The findings in the current setting confirm that safety attitude is a critical individual factor relevant in preventing hazardous behaviour and catastrophic consequences.

We found that safety attitudes were associated with specific attitudes towards the reporting system. Reporting attitudes may be of particular interest, as safety management systems often rely on accurate and honest reporting of incidents and near-misses, and use reports of accidents, nearaccidents and unsafe conditions to assess the safety level of a workplace. However, such an approach is compromised if the workers' reporting is not a reliable indicator. The reporting attitude may be negatively impacted if the individual crew members typically have few incentives for reporting and may fear negative effects or doubt the efficiency of reporting. We will further explore determinants and effects of reporting attitudes in additional datasets (see pre-registrations Sætrevik \& Hjellvik, 2017; Sætrevik \& Hjellvik, 2019).

\section{3: Implications of Findings}

4.3.1: Safety on chemical tanker vessels. The current approach for measuring safety climate, AL, SA and attitudes appear to be applicable for safety on chemical tanker vessels, and comparable high-risk industries. This suggests that the current measures may be used to monitor changes over time, or to identify organizational units with safety indicators that deviates from the average. Comparing the current results with those from vessels in the more heavily regulated hydrocarbon production industry (as seen in Sætrevik, Ørbeck, Helland, \& Eid, 2019) did not indicate any particular differences. The chemical tanker vessels had higher average scores on all the variables, except for "Safety management systems". As mentioned above, a social desirability bias may have contributed to the positive evaluation of safety in the current dataset, but one should note that such biases may be present in all studies using self-reports.

The survey's measures of safety behaviour and how the safety management system is used may be particularly relevant for evaluating risk for accidents. Even minor unsafe actions may have severe consequences on chemical tanker vessels, and how the crew perform their work may be an important predictor of accidents. The safety management system is multifaceted and cover a range of areas that may cause unwanted incidents and accidents. It is reasonable to assume that the crew's behaviour could be a proximal human barrier or cause for dangerous situations. We will further pursue this issue in a follow-up study where we will investigate whether our suggested outcome measures have an impact on the registration of actual unwanted incidents, nearmisses and accidents (see pre-registration Sætrevik \& Hjellvik, 2019). The current study has revealed antecedents of these safety critical outcomes and comparing self-reports with objective data will increase the validity of the current approach. This could be relevant information for enacting procedures, regulations and organizational policies.

4.3.2: Suggested organizational safety interventions. Safety management is a continuous process that requires knowledge about system ergonomics such as contextual relationships, employee competence and experience, as well as information about work tasks and how they are executed (Wilson, 2014). To give priority to safety may imply arranging regular safetyawareness activities, providing for safety even when it competes with commercial interests and providing leaders and safety officers with the necessary conditions to implement the company policy. Thus, the ship-owning companies should encourage the captain to emphasize the importance of following safety regulations when interacting with the crew and make it clear for captains and crew that safety should be maintained even if it comes at the expense of efficiency. The current results showed that the captain's AL was associated with the crew's perception of safety climate. This indicates that an honest, democratic and supportive way of leading may incentivize and reinforce the crew's perceptions, which could further shape attitudes and provide safety benefits. Organizations may want to implement proactive preventive measures directed at improving attitudes, for example through emphasizing the role of safety procedures and regulations in preventing accidents.

\section{4: Evaluation of Study}

The current study was done as a cross-sectional selfreport survey, which makes it subject to influences of "common method variance" (Spector \& Brannick, 2010). As our study consists of only one type of measure made at a single time point, it is difficult to know if the observed relationships could be caused by confounding mechanisms. For example, crew members who were in a good mood on the day of answering the survey, may give optimistic answers throughout the survey. Further, a social desirability bias may cause crew to provide the answers they think the management wants to see. Such effects may be particularly evident when the respondents think that their employer might read their answers (Donaldson \& Grant-Vallone, 2002). While we attempted to assure our participants that captain and company management would not have access to their individual responses, it is unclear how well we succeeded in this. As mentioned above, it is also possible that the crew's cultural frame of reference had an impact on the interpretation of items in the survey. However, similar threats to validity may be present in most studies using self-report measures as various individual, contextual and cultural factors may impact the respondents' answers.

The crew's frame of reference could also impact the extent to which they can accurately report their SA. This means that there could be a difference between individual self-reported answers and actual objective behaviour. Participants may overestimate their SA as they are not aware of elements that could reduce it (Endsley, 1994). We should also mention that the majority of SA research measures the concept in relation to a specific work task or workenvironment, while we currently measure SA across all of a crew members' work tasks (following Sætrevik, 2013). The current study uses the ALQ-scale developed by Walumbwa and colleagues (2008). It should be noted that Walumbwa's research practices have recently been criticized (Marcus, 2014), and some of his findings have been questioned. We nevertheless use the ALQ scale as an example of a leadership 
style that promotes positive individual and interpersonal capacities.

The survey was in English, which is a second language to most of the crew. Item text was rephrased to fit the setting, but manual inspection of completed surveys indicated that some items may nevertheless have been misunderstood. Participants who had clearly misunderstood the surveys (i.e. insensitivity to reversed phrasing of items) were excluded as described above (section 2.1.1). But some may have been uncertain about negatively phrased items and provided answers in the intermediate range, which are more difficult to exclude. As previously mentioned, this may have had an impact on the association between "SA" and "Safe behaviour".

\section{5: Conclusions and Further Research}

The current study has revealed that organizational factors predict safety attitudes which predict safety behaviour and compliance. It shows how the company management and captain's actions impact the attitudes and cognitions of the crew, and that attitudes are critical in reducing risks. For further work in this setting we have pre-registered a followup study (Sætrevik \& Hjellvik, 2019) where we will compare current measurement with the company's record of "nearmisses" and accidents in the year following the survey measure. This will allow us to control for some of the limitations of self-report measures, and to investigate whether the current measures can predict the occurrence of unwanted incidents and accidents.

\section{Acknowledgements}

We appreciate the assistance from the Utkilen ship-owning company, and particular from Roy Håre for advice on item phrasing and assistance in the data collection. Sigurd W. Hystad assisted us in the statistical analyses.

The data collection for the current study was done as a master thesis by authors LRH and REHA under supervision of BS (http://bora.uib.no/handle/1956/17770). The master thesis analysis was pre-registered separately to the analysis presented here (https://osf.io/497v2, both registered before any analysis had been done). The master thesis analysis tested effects of safety climate, AL and SA on unsafe actions, and thus represents a subset of the analysis in the current article.

\section{References}

Ajzen, I. (1991). The theory of planned behavior. Organizational Behavior and Human Decision Processes, 50(2), 179-211. doi:10.1016/0749-5978(91)90020-T

Ajzen, I., \& Fishbein, M. (1973). Attitudinal and normative variables as predictors of specific behavior. Journal of Personality and Social Psychology, 27(1), 41-57. doi: $10.1037 / \mathrm{h} 0034440$

Borgersen, H. C., Hystad, S. W., Larsson, G., \& Eid, J. (2014). Authentic Leadership and Safety Climate Among Seafarers. Journal of Leadership \& Organizational Studies, 21(4), 394-402. doi:10.1177/1548051813499612

Celik, M. (2010). Enhancement of occupational health and safety requirements in chemical tanker operations: The case of cargo explosion. Safety Science, 48(2), 195-203. doi:https://doi.org/10.1016/j.ssci.2009.08.004

Černe, M., Dimovski, V., Marič, M., Penger, S., \& Škerlavaj, M. (2014). Congruence of leader self-perceptions and follower perceptions of authentic leadership: Understanding what authentic leadership is and how it enhances employees' job satisfaction. Australian Journal of Management, 39(3), 453-471. doi: $10.1177 / 0312896213503665$

Clarke, S. (2006). The Relationship Between Safety Climate and Safety Performance: A Meta-Analytic Review. Journal of Occupational Health Psychology, 11(4), 315-327. doi:10.1037/1076-8998.11.4.315
Crano, W. D., \& Prislin, R. (2006). Attitudes and Persuasion. Annual Review of Psychology, 57(1), 345-374. doi:10.1146/annurev.psych.57.102904.190034

Dahl, Ø., Fenstad, J., \& Kongsvik, T. (2014). Antecedents of safety-compliant behaviour on offshore service vessels: a multi-factorial approach. Maritime Policy \& Management, 41(1), 20-41. doi:10.1080/03088839.2013.780311

Dekker, S. W. A., Hummerdal, D. H., \& Smith, K. (2010). Situation awareness: some remaining questions. Theoretical Issues in Ergonomics Science, 11(1-2), 131 135. doi:10.1080/14639220903010092

Donaldson, S., \& Grant-Vallone, E. (2002). Understanding Self-Report Bias in Organizational Behavior Research. Journal of Business and Psychology, 17(2), 245260. doi:10.1023/A:1019637632584

Edgar, G. K., Catherwood, D., Baker, S., Sallis, G., Bertels, M., Edgar, H. E., . . Whelan, A. (2018). Quantitative Analysis of Situation Awareness (QASA): modelling and measuring situation awareness using signal detection theory. Ergonomics, 61(6), 762-777. doi:10.1080/00140139.2017.1420238

Edwards, A. L. (1953). The relationship between the judged desirability of a trait and the probability that the trait will be endorsed. Journal of Applied Psychology, 37(2), 90-93. doi:10.1037/h0058073

Endsley, M. R. (1994). Situation Awareness in Dynamic Human Decision Making: Measurement. In R. D. Gilson, D. J. Garland, \& J. M. Koonce (Eds.), Situational Awareness in Complex Systems (p. 79-97). Daytona Beach: Embry Riddle Aeronautical University Press.

Endsley, M. R. (1995). Toward a Theory of Situation Awareness in Dynamic Systems. Human Factors: The Journal of Human Factors and Ergonomics Society, 37(1), 32-64. doi:10.1518/001872095779049543

Endsley, M. R. (2000). Situation Models: An Avenue to the Modeling of Mental Models. Proceedings of the Human Factors and Ergonomics Society Annual Meeting, 44(1), 61-64. doi:10.1177/154193120004400117

Flin, R., Mearns, K., O'Connor, P., \& Bryden, R. (2000). Measuring safety climate: identifying the common features. Safety Science, 34(1-3), 177-192. doi:10.1016/S0925-7535(00)00012-6

Gardner, W. L., Avolio, B. J., Luthans, F., May, D. R., \& Walumbwa, F. (2005) "Can you see the real me?" A self-based model of authentic leader and follower development. The Leadership Quarterly, 16(3), 343-372. doi:10.1016/j.leaqua.2005.03.003

Guldenmund, F. W. (2000). The nature of safety culture: a review of theory and research. Safety Science, 34(1-3), 215-257. doi:10.1016/S0925-7535(00)00014-X

Hobbs, A., \& Williamson, A. (2002). Unsafe acts and unsafe outcomes in aircraft maintenance. Ergonomics, 45(12), 866-882. doi:10.1080/00140130210148528

Hofmann, D. A., \& Stetzer, A. (1996). A cross-level investigation of factors influencing unsafe behaviors and accidents. Personnel Psychology, 49(2), $307-$ 339.

Hofstede, G. (2011). Dimensionalizing Cultures: The Hofstede Model in Context. Online Readings in Psychology and Culture. 2, 1. doi:10.9707/23070919.1014

Hu, L.-T., \& Bentler, P. M. (1999). Cutoff criteria for fit indexes in covariance structure analysis: Conventional criteria versus new alternatives. Structural Equation Modeling: A Multidisciplinary Journal, 6(1), 1-55. doi:10.1080/10705519909540118

Hystad, S. W., Bartone, P. T., \& Eid, J. (2014). Positive organizational behavior and safety in the offshore oil industry: Exploring the determinants of positive safety climate. The Journal of Positive Psychology, 9(1), 42-53. doi:10.1080/17439760.2013.831467

Lappalainen, J., Vepsäläinen, A., Salmi, K., \& Tapaninen, U. (2011). Incident reporting in Finnish shipping companies. The international Journal for professionals in maritime administration, industry and education, 10(2), 167-181. doi:10.1007/s13437-011-0011-0

Lu, C.-S., \& Tsai, C.-L. (2010). The effect of safety climate on seafarers' safety behaviors in container shipping. Accident Analysis and Prevention, 42(6), 1999 2006. doi:10.1016/j.aap.2010.06.008

Marcus, A. (2014). Univ.: No misconduct, but "poor research practice" in mgt prof's work now subject to 7 retractions. Retrieved from https://retractionwatch.com/2014/11/14/univ-no-misconduct-but-poorresearch-practice-in-mgt-profs-work-now-subject-to-7-retractions/\#more23666

Mearns, K., \& Flin, R. (1995). Risk perception and attitudes to safety by personnel in the offshore oil and gas industry: a review. Journal of Loss Prevention in the Process Industries, 8(5), 299-305. doi:10.1016/0950-4230(95)00032-V

Mearns, K., Flin, R., Gordon, R., \& Fleming, M. (1998). Measuring safety climate on offshore installations. Work \& Stress, 12(3), 238-254. doi:10.1080/02678379808256864

Nielsen, M. B., Eid, J., Hystad, S., Saetrevik, B., \& Saus, E. (2013). A brief safety climate inventory for petro-maritime organizations. Safety Science, 58, 81-88. doi:10.1016/j.ssci.2013.04.002

Norwegian Maritime Authority. (2017). Focus on risks 2018. Retrieved from https://www.sdir.no/globalassets/sjofartsdirektoratet/fartoy-og-sjofolk--dokumenter/roh---dokumenter/focus-on-risk-2018.pdf?t=1572517338858

Probst, T. M., \& Graso, M. (2013). Pressure to produce $=$ pressure to reduce accident reporting? Accident Analysis and Prevention, 59, 580-587. doi:10.1016/j.aap.2013.07.020

Reason, J. (1990). Human error: Cambridge university press. 
Roberts, S. E. (2002). Hazardous occupations in Great Britain. The Lancet, 360(9332), 543-544. doi:10.1016/S0140-6736(02)09708-8

Rundmo, T. (1994). Associations between safety and contingency measures and occupational accidents on offshore petroleum platforms. Scandinavian Journal of Work Environment \& Health, 20(2), 128-131.

Salmon, P., Stanton, N., Walker, G., \& Green, D. (2006). Situation awareness measurement: A review of applicability for $\mathrm{C} 4 \mathrm{i}$ environments. Applied Ergonomics, 37(2), 225-238. doi:10.1016/j.apergo.2005.02.001

Salmon, P. M., Stanton, N. A., Walker, G. H., Jenkins, D., Ladva, D., Rafferty, L., \& Young, M. (2009). Measuring Situation Awareness in complex systems: Comparison of measures study. International Journal of Industrial Ergonomics, 39(3), 490-500. doi:10.1016/j.ergon.2008.10.010

Sandhåland, H., Oltedal, H. A., Hystad, S. W., \& Eid, J. (2017). Effects of leadership style and psychological job demands on situation awareness and the willingness to take a risk: A survey of selected offshore vessels. Safety Science, 93, 178-186. doi:10.1016/j.ssci.2016.12.004

Sneddon, A., Mearns, K., \& Flin, R. (2006). Situation awareness and safety in offshore drill crews. Cognition, Technology \& Work, 8(4), 255-267. doi:10.1007/s10111-006-0040-1

Spector, P. E., \& Brannick, M. T. (2010). Common Method Issues: An Introduction to the Feature Topic in Organizational Research Methods. Organizational Research Methods, 13(3), 403-406. doi: $10.1177 / 1094428110366303$

Storgård, J., Erdogan, I., Lappalainen, J., \& Tapaninen, U. (2012). Developing Incident and Near Miss Reporting in the Maritime Industry-A Case Study on the Baltic Sea. Procedia - Social and Behavioral Sciences, 48(C), 1010-1021. doi:10.1016/j.sbspro.2012.06.1078

Sætrevik, B. (2013). Developing a context-general self-report approach to measure three-level situation awareness. International maritime health, 64(2), 66-71.

Sætrevik, B., \& Hjellvik, L. R. (2017). Reporting attitudes and determinants of incident reporting on maritime vessels. Retrieved from osf.io/z6gdq

Sætrevik, B., \& Hjellvik, L. R. (2019). Association of individual safety variables with objective safety outcomes. Retrieved from osf.io/cj8fr

Sætrevik, B., \& Hystad, S. W. (2017). Situation awareness as a determinant for unsafe actions and subjective risk assessment on offshore attendant vessels. Safety Science, 93, 214-221. doi:10.1016/j.ssci.2016.12.012

Sætrevik, B., \& Hystad, S. W. (2019). Ship leadership, situation awareness and crew safety behavior - Pre-registered replications in two survey datasets. Retrieved from https://doi.org/10.31219/osf.io/y6jsa

Sætrevik, B., Ørbeck, S. B., Helland, M. V., \& Eid, J. (2019). Decreased job security without change in safety during hydrocarbon industry recession. Retrieved from https://doi.org/10.31234/osf.io/8xeg5

Tomás, J. M., Cheyne, A., \& Oliver, A. (2011). The Relationship Between Safety Attitudes and Occupational Accidents: The Role of Safety Climate. European Psychologist, 16(3), 209-219. doi:10.1027/1016-9040/a000036

Törner, M. (2011). The "social-physiology" of safety. An integrative approach to understanding organisational psychological mechanisms behind safety performance. Safety Science, 49(8), 1262-1269. doi:10.1016/j.ssci.2011.04.013

Wagenmakers, E.-J., Wetzels, R., Borsboom, D., van Der Maas, H. L. J., \& Kievit, R. A. (2012). An Agenda for Purely Confirmatory Research. Perspectives on Psychological Science, 7(6), 632-638. doi:10.1177/1745691612463078

Walumbwa, F. O., Avolio, B. J., Gardner, W. L., Wernsing, T. S., \& Peterson, S. J. (2008). Authentic Leadership: Development and Validation of a TheoryBased Measuret. Journal of Management, 34(1), 89-126. doi:10.1177/0149206307308913

Wilson, J. R. (2014). Fundamentals of systems ergonomics/human factors. Applied Ergonomics, 45(1), 5-13. doi:10.1016/j.apergo.2013.03.021

Zohar, D. (1980). Safety climate in industrial organizations: Theoretical and applied implications. Journal of Applied Psychology, 65(1), 96-102. doi:10.1037/00219010.65.1.96

Zohar, D. (2000). A Group-Level Model of Safety Climate: Testing the Effect of Group Climate on Microaccidents in Manufacturing Jobs. Journal of Applied Psychology, 85(4), 587-596. doi:10.1037/0021-9010.85.4.587

Zohar, D. (2010). Thirty years of safety climate research: Reflections and future directions. Accident Analysis \& Prevention, 42(5), 1517-1522. doi:https://doi.org/10.1016/j.aap.2009.12.019

Zohar, D., \& Luria, G. (2005). A Multilevel Model of Safety Climate: Cross-Level Relationships Between Organization and Group-Level Climates. Journal of Applied Psychology, 90(4), 616-628. doi:10.1037/0021-9010.90.4.616

Zsambok, E., C. (2014). Naturalistic Decision Making: Where Are We Now? . In C.E. Zsambok \& G. Klein (Eds.), Naturalistic Decision Making (p. 3-16). New York: Psychology Press. 